\title{
DRAZIN INVERSE OF SINGULAR ADJACENCY MATRICES OF DIRECTED WEIGHTED CYCLES
}

\author{
ANDRÉS M. ENCINAS, DANIEL A. JAUME, CRISTIAN PANELO, \\ AND ADRIÁN PASTINE
}

\begin{abstract}
We present a necessary and sufficient condition for the singularity of circulant matrices associated with directed weighted cycles. This condition is simple and independent of the order of matrices from a complexity point of view. We give explicit and simple formulas for the Drazin inverse of these circulant matrices. We also provide a Bjerhammar-type condition for the Drazin inverse.
\end{abstract}

\section{INTRODUCTION AND PRELIMINARIES}

Circulant matrices appear in many applications, for example to approximate the finite difference of elliptic equations with periodic boundary (see [8]) and to approximate periodic functions with splines (see [9]); they also play an important role in coding theory and in statistics. The standard references are [10] and [13].

Direct computation methods for the inverse of some circulant matrices have been proposed in many works: see for example [22], [26], [12], [23], [27, [16, [7], and [19] (we order the papers chronologically). Singular circulant matrices have received less attention: see [3] and [25].

Graphs whose adjacency matrix is circulant, specially those with integral spectrum, have been studied in several articles: see for example [24], [2], [14], [18, and 21.

In [15] a combinatorial description of the Drazin inverse of the adjacency matrix of a tree is exhibited. In the present work we study the Drazin inverse of the singular adjacency matrix of directed weighted cycles, as a first step in order to give a combinatorial description of the Drazin inverse of other graphs. The standard reference for generalized inverses is [4. For all linear algebra-theoretic notions not

2010 Mathematics Subject Classification. Primary 15A09, 05C38.

Key words and phrases. Circulant matrix; Drazin inverse; Directed weighted cycles.

Adrián Pastine, Cristian Panelo, and Daniel A. Jaume have been partially supported by Universidad Nacional de San Luis, grant PROICO 03-0918, and by MATH AmSud, grant 18-MATH-01. Daniel A. Jaume has been partially supported by "Programa de becas de integración regional para argentinos", grant 2075/2017. Andrés M. Encinas has been partially supported by Comisión Interministerial de Ciencia y Tecnología under projects MTM2014-60450-R and MTM2017-85996-R. 
defined here, the reader is referred to [17]; for all graph-theoretic notions not defined here, the reader is referred to [11.

This work is organized as follows. In Section 2 we give a Bjerhammar-type condition for the Drazin inverse. In the setting of Moore-Penrose theory, Bjerhammar, in [5], was the first to notice that if you have additional information about the fundamental spaces of the matrix and its candidate to generalized inverse you do not need to check all the Moore-Penrose conditions. In Section 3 we give some basic properties of circulant matrices. In Section 4 we characterize which circulant matrices associated to directed cycles are singular. In Sections 5, 6, and 7 we give the Drazin inverse of singular circulant matrices associated with cycles.

Throughout the paper, given $a \in \mathbb{C}$ we denote by $a^{\#}$ the number

$$
a^{\#}= \begin{cases}a^{-1}, & \text { if } a \neq 0 \\ 0, & \text { if } a=0 .\end{cases}
$$

Let $A=\left(a_{i j}\right)$ be a matrix of order $n$. All the matrices in this work have real or complex coefficients. We associate with $A$ a digraph $D(A)$ with $n$ vertices. The vertices of $D(A)$ are denoted by $0,1,2, \ldots, n-1$. There is an edge from vertex $i$ to vertex $j$ of weight $a_{i j}$ for each $i, j=0,1,2, \ldots, n-1$. A linear subdigraph of $D(A)$ is a spanning subdigraph of $D(A)$ in which each vertex has indegree 1 and outdegree 1 . We associate to $A$ a second digraph $D^{*}(A)=D\left(A^{T}\right)$, where $A^{T}$ is the transpose of $A$. This digraph is called the Coates digraph of the matrix $A$.

Theorem 1.1 ([6, Definition 4.1.1 and Theorem 4.2.11]). Let $A=\left(a_{i j}\right)$ be a square matrix of order $n$. Then

$$
\operatorname{det}(A)=\sum_{L \in \mathcal{L}\left(D^{*}(A)\right)}(-1)^{n-c(L)} w(L),
$$

where $\mathcal{L}\left(D^{*}(A)\right)$ is the set of all linear subdigraphs of the Coates digraph of $A, c(L)$ is the number of cycles contained in $L$, and $w(L)$ is the product of the weights of the edges of $L$.

\section{BJERHAMMAR-TYPE CONDITION FOR THE DRAZIN INVERSE}

Given a matrix $A$ the column space of $A$ is denoted by $\mathcal{R}(A)$ and its dimension by $\operatorname{rank}(A)$. The null space of $A$ is denoted by $\mathcal{N}(A)$ and its dimension, called nullity, by $\operatorname{null}(A)$.

Definition $2.1(4)$. The index of a square matrix $A$, denoted by $\operatorname{Ind}(A)$, is the smallest nonnegative integer $k$ for which $\mathcal{R}\left(A^{k}\right)=\mathcal{R}\left(A^{k+1}\right)$.

A matrix is nonsingular if and only if it has index 0 .

Definition 2.2 (4 $)$. Let $A$ be a matrix of index $k$. The Drazin inverse of $A$, denoted by $A^{D}$, is the unique matrix such that

(1) $A A^{D}=A^{D} A$,

(2) $A^{k+1} A^{D}=A^{k}$

(3) $A^{D} A A^{D}=A^{D}$. 
Note that the Drazin inverse of a matrix of index 1 equals its group inverse (that is denoted, for a matrix $A$, by $A^{\#}$ ); see [4].

We prove a Bjerhammar-type condition for the Drazin inverse (see [4]). We do this because, for some combinatorial problems, Condition 3 in Definition 2.2 is more difficult to verify (directly) than Conditions 1 and 2. Theorem 2.4 is a generalization of Theorem 8.3 in [15] and it is usually more suitable for combinatorial problems.

Lemma 2.3. Let $A$ and $D$ be square matrices of order $n$, with $\operatorname{Ind}(A)=k$, such that $\mathcal{N}\left(A^{k}\right)=\mathcal{N}(D)$ and $A D=D A$. Then $\mathcal{R}\left(A^{k}\right)=\mathcal{R}(D)$.

Proof. Since $\mathcal{N}\left(A^{k}\right)=\mathcal{N}(D)$, we have that $\operatorname{rank}\left(A^{k}\right)=\operatorname{rank}(D)$. If we prove that $\mathcal{R}(D) \subset \mathcal{R}\left(A^{k}\right)$, then $\mathcal{R}(D)=\mathcal{R}\left(A^{k}\right)$.

The key observation is that $\mathcal{R}(D)=\mathcal{R}\left(D A^{k}\right)$. Which, together with the fact that $A D=D A$ implies $A^{k} D=D A^{k}$, gives us $\mathcal{R}(D)=\mathcal{R}\left(A^{k} D\right) \subset \mathcal{R}\left(A^{k}\right)$.

In order to prove that $\mathcal{R}(D)=\mathcal{R}\left(D A^{k}\right)$, note that

$$
\begin{aligned}
\mathcal{R}(D) & =D\left(\mathcal{R}\left(A^{k}\right) \oplus \mathcal{N}\left(A^{k}\right)\right) \\
& =D\left(\mathcal{R}\left(A^{k}\right)\right) \\
& =\mathcal{R}\left(D A^{k}\right) .
\end{aligned}
$$

Theorem 2.4. Let $A$ and $D$ be square matrices of order $n$, with $\operatorname{Ind}(A)=k$, such that $\mathcal{N}\left(A^{k}\right)=\mathcal{N}(D)$ and $A D=D A$. Then $A^{k+1} D=A^{k}$ if and only if $D^{2} A=D$.

Proof. Let $A$ and $B$ be two square matrices of order $n$. We use the fact that $A=B$ if and only if $A \vec{x}=B \vec{x}$ for every vector $\vec{x} \in \mathbb{C}^{n}$. It is well known that $\mathbb{C}^{n}=\mathcal{R}\left(A^{k}\right) \oplus \mathcal{N}\left(A^{k}\right)$, where $k$ is the index of $A$ (see [17]). Therefore, we can check that $A \vec{x}=B \vec{x}$ for every vector $\vec{x} \in \mathbb{C}^{n}$ by checking that $A \vec{z}=B \vec{z}$ for all $\vec{z} \in \mathcal{R}\left(A^{k}\right)$ and $A \vec{x}=B \vec{x}$ for all $\vec{x} \in \mathcal{N}\left(A^{k}\right)$.

Assume $A^{k+1} D=A^{k}$. Let $\vec{x} \in \mathcal{N}\left(A^{k}\right)$. Hence, $\vec{x} \in \mathcal{N}(D)$. Therefore $D^{2} A \vec{x}=$ $A D^{2} \vec{x}=\overrightarrow{0}=D \vec{x}$. Let $\vec{z} \in \mathcal{R}\left(A^{k}\right)$. Then there exists a vector $\vec{y}$ such that $\vec{z}=A^{k} \vec{y}$. Therefore $D \vec{z}=D A^{k} \vec{y}=D^{2} A^{k+1} \vec{y}=D^{2} A A^{k} \vec{y}=D^{2} A \vec{z}$.

Assume now $D^{2} A=D$. Let $\vec{x} \in \mathcal{N}\left(A^{k}\right)$. Then $A^{k+1} D \vec{x}=D A^{k+1} \vec{x}=\overrightarrow{0}=A^{k} \vec{x}$. Let $\vec{z} \in \mathcal{R}\left(A^{k}\right)$. Then by Lemma $2.3 \vec{z} \in \mathcal{R}(D)$. Thus, there exists a vector $\vec{y}$ such that $\vec{z}=D \vec{y}$. Hence, $A^{k} \vec{z}=A^{k} D \vec{y}=A^{k} D^{2} A \vec{y}=A^{k+1} D^{2} \vec{y}=A^{k+1} D \vec{z}$.

\section{Some PRoperties of CIRCUlant MATRICES}

Definition 3.1. An $n \times n$ matrix $A=\left(a_{i j}\right)$ is a circulant matrix if it has the form $a_{i j}=\alpha_{j-i}$ for some $\alpha_{0}, \alpha_{1}, \ldots, \alpha_{n-1}$, where the subscript $j-i$ is taken modulo $n$. We denote

$$
A=\operatorname{Circ}\left(\alpha_{0}, \alpha_{1}, \ldots, \alpha_{n-1}\right)=\left[\begin{array}{cccc}
\alpha_{0} & \alpha_{1} & \cdots & \alpha_{n-1} \\
\alpha_{n-1} & \alpha_{0} & \cdots & \alpha_{n-2} \\
\vdots & \vdots & \ddots & \vdots \\
\alpha_{1} & \alpha_{2} & \cdots & \alpha_{0}
\end{array}\right]
$$


Note that $\operatorname{Circ}\left(\alpha_{0}, \alpha_{1}, \ldots, \alpha_{n-1}\right)^{T}=\operatorname{Circ}\left(\alpha_{0}, \alpha_{n-1}, \ldots, \alpha_{1}\right)$. Many properties about circulant matrices are well known. For instance, if we consider

$$
A=\operatorname{Circ}\left(\alpha_{0}, \alpha_{1}, \ldots, \alpha_{n-1}\right),
$$

then $A$ can be expressed in the form

$$
C=\sum_{k=0}^{n-1} \alpha_{k} P^{k},
$$

where $P$ is the cyclic permutation matrix of order $n$; that is,

$$
P^{k}=\left(\begin{array}{cc}
O_{n-k, k} & I_{n-k} \\
I_{k} & O_{k, n-k}
\end{array}\right),
$$

where $k \in\{1, \ldots, n-1\}, I_{n}$ is the identity matrix of order $n, P^{0}=I_{n}$, and $O_{m, n}$ is an $m \times n$ zero-matrix (see [10]).

In addition, circulant matrices of order $n$ form an $n$-dimensional vector space and also a commutative algebra, since for any two given circulant matrices its product is also a circulant matrix, and moreover any two circulant matrices commute with each other. Therefore, any circulant matrix is normal, which implies that it has index 1, and therefore any circulant matrix has group inverse that coincides with its Moore-Penrose inverse. Although not every generalized inverse of a circulant matrix must be circulant itself, this property holds for the group inverse.

One of the main problems in the field of circulant matrices is to determine the Drazin (group) inverse of a circulant matrix, and moreover to know when the matrix is in fact invertible.

This problem has been widely studied in the literature by using the primitive $n$-th root of unity and some polynomial associated with it (see [13] and [23]). Specifically, let $\omega=e^{\frac{2 \pi}{n} i}$ be the primitive $n$-th root of unity. In addition, define for each $j=0, \ldots, n-1$, the vector $\vec{t}_{j}=\left(1, \omega^{j}, \ldots, \omega^{j(n-1)}\right)^{T} \in \mathbb{R}^{n}$ and for any $\vec{a}=\left(\alpha_{0}, \ldots, \alpha_{n-1}\right)^{T} \in \mathbb{R}^{n}$ the polynomial $P_{\vec{a}}(x)=\sum_{j=0}^{n-1} \alpha_{j} x^{j}$. Observe that $\vec{t}_{0}=(1, \ldots, 1)^{T}$. The following lemma provides a necessary and sufficient condition for the invertibility of $\operatorname{Circ}\left(\alpha_{0}, \ldots, \alpha_{n-1}\right)$ and gives a formula for its group inverse (see [10]).

Lemma 3.2. For any $\left(\alpha_{0}, \ldots, \alpha_{n-1}\right)^{T} \in \mathbb{R}^{n}$, the following properties hold:

(i) $\operatorname{Circ}\left(\alpha_{0}, \ldots, \alpha_{n-1}\right) \vec{t}_{j}=P_{\vec{a}}\left(\omega^{j}\right) \vec{t}_{j}$, for any $j=0, \ldots, n-1$. In particular, $\operatorname{det} \operatorname{Circ}\left(\alpha_{0}, \ldots, \alpha_{n-1}\right)=\prod_{k=0}^{n-1} P_{\vec{a}}\left(\omega^{k}\right)$ and hence, $\operatorname{Circ}\left(\alpha_{0}, \ldots, \alpha_{n-1}\right)$ is invertible if and only if $P_{\vec{a}}\left(\omega^{j}\right) \neq 0, j=0, \ldots, n-1$.

(ii) $\operatorname{Circ}\left(\alpha_{0}, \ldots, \alpha_{n-1}\right)^{\#}=\operatorname{Circ}\left(\beta_{0}, \ldots, \beta_{n-1}\right)$, where, for $j=0, \ldots, n-1$,

$$
\beta_{j}=\frac{1}{n} \sum_{k=0}^{n-1} \omega^{-k j} P_{\vec{a}}\left(\omega^{k}\right)^{\#} .
$$


On one hand, let us notice that property (i) of the previous lemma implies that all circulant matrices of order $n$ have the same eigenvectors but different eigenvalues. On the other hand, part (ii) in the above lemma establishes that the problem of finding the Drazin (group) inverse of a circulant matrix is completely solved. However, the computational complexity of formula (ii) for the determination of its group inverse grows with the order of the matrix, so it is not useful at all from the computational point of view. So, it is interesting to look for alternative expressions for the group inverse of specific classes of circulant matrices. Over the years, many papers have considered this topic specially for circulant matrices with few nonzero entries. In many of these cases, the special structure of the matrix is highly used and leads to the employment of alternative methods, for example solving linear difference equations (see for instance [7, 20], and [22]). The aim of this work is to provide formulas for the Drazin (group) inverse of a circulant matrix associated with directed weighted cycles.

\section{Circulant matrices with two parameters}

Circulant matrices of the form $\operatorname{Circ}(0, a, 0, \ldots, 0, b)$ of order $n$ are denoted by $\mathrm{C}_{\mathrm{n}}(a, b)$. The next theorem gives a simple and independent of the order, necessary and sufficient condition for the singularity of $\mathrm{C}_{\mathrm{n}}(a, b)$. We usually call the Coates digraph of $\mathrm{C}_{\mathrm{n}}(a, b)$ "cycle" or "directed cycle".

Theorem 4.1. $\mathrm{C}_{\mathrm{n}}(a, b)$ is singular if and only if either $b=-a$ for all $n$ or $b=a$ and $n=0 \bmod 4$.

Proof. We break the proof in three parts.

If $n=0 \bmod 4$, then the Coates digraph $D^{*}\left(\mathrm{C}_{4 k}(a, b)\right)$, where $k$ is some positive integer, looks like the digraph in Figure 1 and has four linear subdigraphs (see Figure 2. Hence, by Theorem 1.1.

$$
\operatorname{det}\left(\mathrm{C}_{4 k}(a, b)\right)=-\left(a^{2 k}-b^{2 k}\right)^{2} .
$$

Therefore, $\operatorname{det}\left(\mathrm{C}_{4 k}(a, b)\right)=0$ if and only if $|a|=|b|$.

If $n=2 k+1$ with $k \in \mathbb{N}$, then the Coates digraph $D^{*}\left(\mathrm{C}_{2 k+1}(a, b)\right)$ looks like in Figure 3 Therefore, the cycles of length $2 k+1$ have two linear subdigraphs (see Figure 4. Hence, by Theorem 1.1 .

$$
\operatorname{det}\left(C_{2 k+1}(a, b)\right)=a^{2 k+1}+b^{2 k+1} \text {. }
$$

Therefore, $\operatorname{det}\left(\mathrm{C}_{2 k+1}(a, b)\right)=0$ if and only if $a=-b$.

If $n=4 k+2$ with $k \in \mathbb{N}$, then the Coates digraph $D^{*}\left(\mathrm{C}_{4 k+2}(a, b)\right)$ has four linear subdigraphs; they look similar to the ones in Figure 2 Hence,

$$
\operatorname{det}\left(\mathrm{C}_{4 k+2}(a, b)\right)=-\left(a^{2 k+1}+b^{2 k+1}\right)^{2} .
$$

Therefore, $\operatorname{det}\left(\mathrm{C}_{4 k+2}(a, b)\right)=0$ if and only if $a=-b$.

Our objective is to give formulas for the Drazin inverse of singular adjacency matrices of cycles. By Theorem 4.1. we need to study three cases: Case 1, when $n=0 \bmod 4$; Case 2, when $n=2 \bmod 4$; and Case 3, when $n=1 \bmod 2$. This will be done in the next three sections, one for each case. 


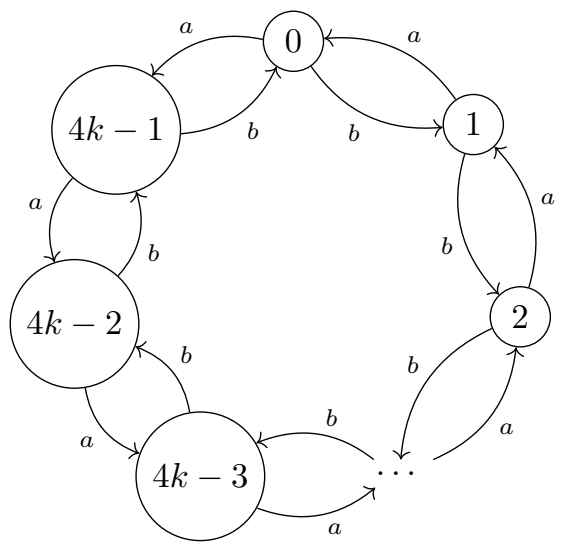

Figure 1. A directed cycle of length $4 k$

5. CASE 1: $n=0 \bmod 4$

As shown in Theorem 4.1. $\mathrm{C}_{4 k}(a, b)$ is singular only if $a=b$ or $a=-b$. In this section we provide formulas for the Drazin inverse in each case. Our proof strategy is the following: We show that a given candidate satisfies the conditions in Definition 2.2 Note that, since we are working with circulant matrices, the commutative property always holds. Thus, we just need to prove Conditions 2 and 3. We prove Condition 2 directly, but Condition 3 is proved via Theorem 2.4 This is the main section of this work. Ideas developed here will be used in the next sections.

Definition 5.1. Let $n=0 \bmod 4$ and $a \in \mathbb{R}$. With $C_{n}^{D 1}(a, a)$ we denote the circulant matrix

$$
\frac{a^{\#}}{n} \operatorname{Circ}\left(\delta_{0}, \delta_{1}, \ldots, \delta_{n-1}\right),
$$

where $\delta_{j}$ is the lowest nonnegative integer such that

$$
\delta_{j}=\left((-1)^{\left\lfloor\frac{j}{2}\right\rfloor}\left\lfloor\frac{n-j}{2}\right\rfloor+(-1)^{\left\lfloor\frac{n-j}{2}\right\rfloor}\left\lfloor\frac{j}{2}\right\rfloor\right) \bmod \left(\frac{n}{2}\right),
$$

for $0 \leq j \leq n-1$.

In order to prove that $\mathrm{C}_{n}^{D 1}(a, a)$ is the Drazin inverse of $\mathrm{C}_{n}(a, a)$ we need the following results.

Lemma 5.2. Let $\delta_{j}$ be as in Definition 5.1. If $j=0 \bmod 2$, then $\delta_{j}=0$.

Proof. Let $t \in \mathbb{N} \cup\{0\}$. Then

$$
\begin{aligned}
\delta_{2 t} & =\left((-1)^{\lfloor t\rfloor}\lfloor(2 k-t)\rfloor+(-1)^{\lfloor(2 k-t)\rfloor}\lfloor t\rfloor\right) \bmod 2 k \\
& =(-1)^{t} 2 k \bmod 2 k \\
& =0 .
\end{aligned}
$$




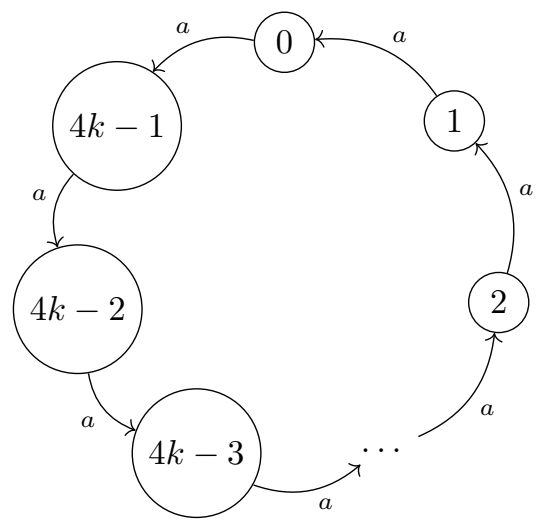

(A) First linear subdigraph of a cycle of length $4 k$
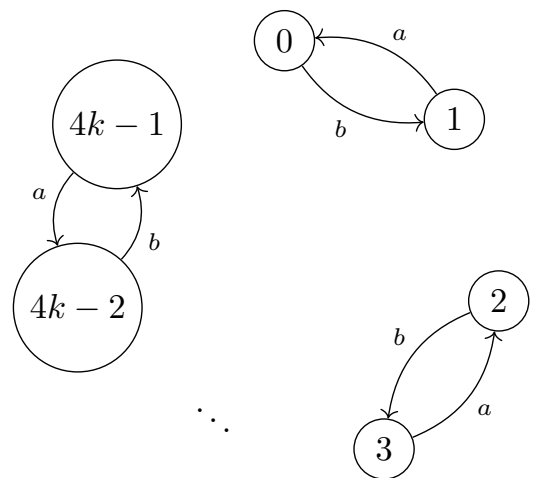

(C) Third linear subdigraph of a cycle of length $4 k$

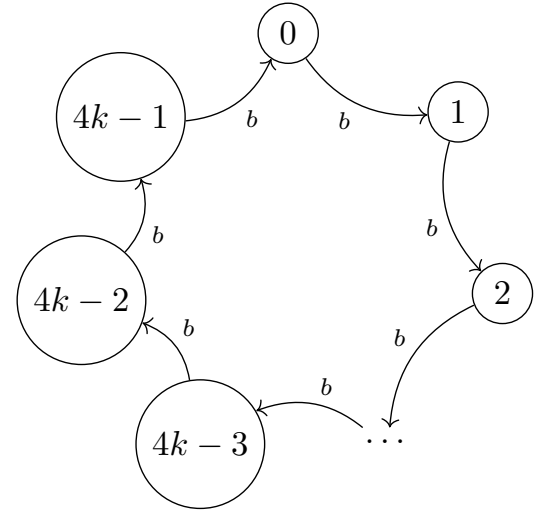

(B) Second linear subdigraph of a cycle of length $4 k$

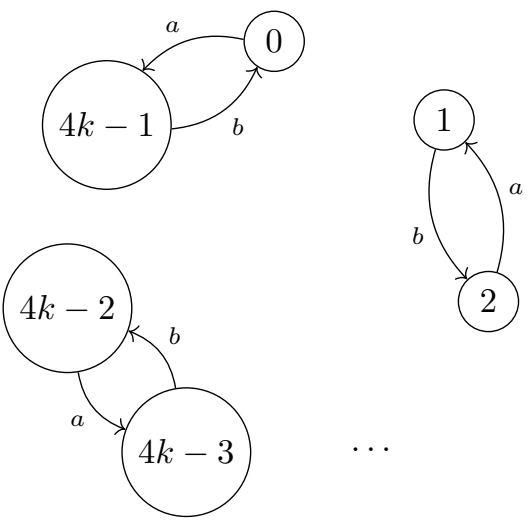

(D) Fourth linear subdigraph of a cycle of length $4 k$

FiguRE 2. The different linear subdigraphs of a cycle of length $4 k$

The next lemma follows directly from Definition 5.1

Lemma 5.3. Let $n=4 k$ and $\delta_{j}$ be as in Definition 5.1. If $j \in \mathbb{N} \cup\{0\}$, then $\delta_{j}=\delta_{4 k-j}$.

The next theorem allows us to prove Condition 3 of the definition of Drazin inverse via Theorem 2.4 Note that both $D\left(\mathrm{C}_{4 k}(a, a)\right)$ and $D\left(\mathrm{C}_{4 k}^{D 1}(a, a)\right)$ are bipartite, with partition into even and odd vertices. This means that there are no directed edges between odd vertices, nor between even vertices.

Theorem 5.4. $\mathcal{N}\left(\mathrm{C}_{4 k}(a, a)\right)=\mathcal{N}\left(\mathrm{C}_{4 k}^{D 1}(a, a)\right)$. 


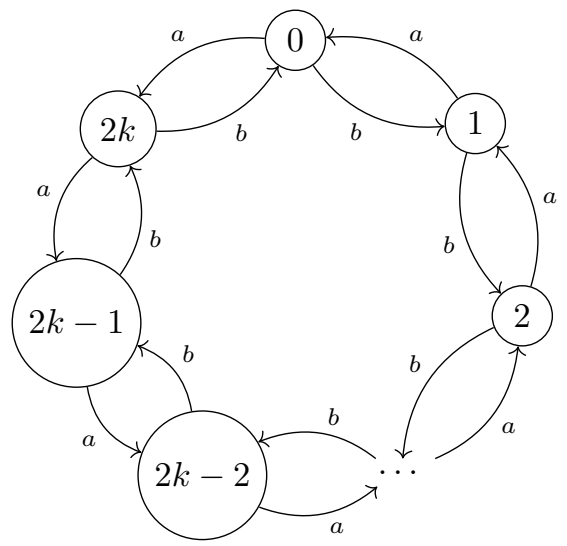

Figure 3. A directed cycle of length $2 k+1$

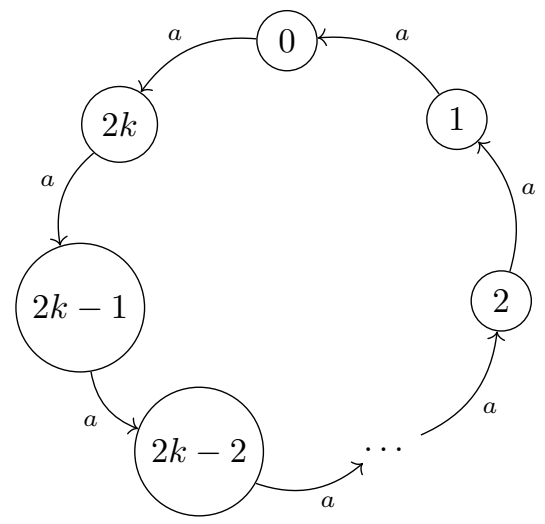

(A) First linear subdigraph of a cycle of length $2 k+1$

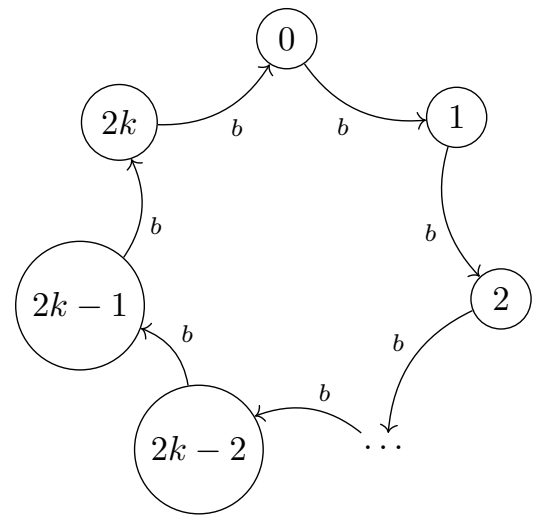

(B) Second linear subdigraph of a cycle of length $2 k+1$

Figure 4. The different linear subdigraphs of a cycle of length $2 k+1$

Proof. Assume $a \neq 0$. By the preceding discussion, writing $\mathrm{C}_{4 k}(a, a)$ in circulant notation:

$$
\mathrm{C}_{4 k}(a, a)=\operatorname{Circ}\left(c_{0}, c_{1}, \ldots, c_{4 k-1}\right) .
$$

Note that $c_{j}=c_{4 k-j}$; this fact is used in Equation (5.1).

In $D\left(\mathrm{C}_{4 k}^{D 1}(a, a)\right)$, if $i=1 \bmod 2$, then the vertex $i$ has directed edges to some even vertices, and if $i=0 \bmod 2$, then the vertex $i$ has directed edges to some odd vertices. See Figure 5 
We take the vectors $\vec{x}=\left(x_{0}, x_{1}, \ldots, x_{4 k-1}\right)^{T}$ and $\vec{y}=\left(y_{0}, y_{1}, \ldots, y_{4 k-1}\right)^{T}$, where

$$
x_{i}=\left\{\begin{aligned}
1, & \text { if } i=0 \bmod 4 ; \\
-1, & \text { if } i=2 \bmod 4 ; \\
0, & \text { otherwise, }
\end{aligned} \quad \text { and } \quad y_{i}=\left\{\begin{aligned}
1, & \text { if } i=1 \bmod 4 \\
-1, & \text { if } i=3 \bmod 4 \\
0, & \text { otherwise. }
\end{aligned}\right.\right.
$$

We prove that both vectors, $\vec{x}$ and $\vec{y}$, are in the null space of both matrices, $\mathrm{C}_{4 k}(a, a)$ and $\mathrm{C}_{4 k}^{D 1}(a, a)$. We do this componentwise.

On one hand, if $i=0 \bmod 2$, then by Lemma 5.3. $\left(\mathrm{C}_{4 k}(a, a) \vec{x}\right)_{i}=0$, and if $i=1$ $\bmod 2$, then

$$
\left(\mathrm{C}_{4 k}(a, a) \vec{x}\right)_{i}=\sum_{j=0}^{4 k-1}\left( \pm c_{j} \mp c_{4 k-j}\right)=0,
$$

where $c_{4 k}:=c_{0}$. This proves that $\vec{x} \in \mathcal{N}\left(\mathrm{C}_{4 k}(a, a)\right)$. Mutatis mutandis, $\vec{y} \in$ $\mathcal{N}\left(\mathrm{C}_{4 k}(a, a)\right)$. On the other hand, if $i=0 \bmod 2$, then the $i$-th coordinate of the vector $\mathrm{C}_{4 k}^{D 1}(a, a) \vec{x}$ is zero, i.e. $\left(\mathrm{C}_{4 k}^{D 1}(a, a) \vec{x}\right)_{i}=0$, and if $i=1 \bmod 2$, then

$$
\left(\mathrm{C}_{4 k}^{D 1}(a, a) \vec{x}\right)_{i}=\sum_{j=0}^{4 k-1}\left( \pm \delta_{j} \mp \delta_{4 k-j}\right)=0,
$$

where $\delta_{4 k}:=\delta_{0}$ and by Lemma $5.3 \delta_{j}:=\delta_{4 k-j}$ for $j \in\{1, \ldots, 4 k-1\}$. This proves that $\vec{x} \in \mathcal{N}\left(\mathrm{C}_{4 k}^{D 1}(a, a)\right)$. Mutatis mutandis, $\vec{y} \in \mathcal{N}\left(\mathrm{C}_{4 k}^{D 1}(a, a)\right)$. Therefore, $\operatorname{null}\left(\mathrm{C}_{4 k}(a, a)\right) \geq 2$ and $\operatorname{null}\left(\mathrm{C}_{4 k}^{D 1}(a, a)\right) \geq 2$. Note that $\mathrm{C}_{4 k}(a, a)(\{4 k-$ $1,4 k\} \mid\{1,4 k\})$, the submatrix of $\mathrm{C}_{4 k}(a, a)$ obtained by deleting the last two rows and the first and last columns, and $\mathrm{C}_{4 k}^{D 1}(a, a)(\{4 k-1,4 k\} \mid\{1,4 k\})$ are nonsingular (we follow the notation given in [1] for submatrices). Hence, $\operatorname{rank}\left(\mathrm{C}_{4 k}(a, a)\right) \geq$ $4 k-2$ and $\operatorname{rank}\left(\mathrm{C}_{4 k}^{D 1}(a, a)\right) \geq 4 k-2$. Therefore,

$$
\operatorname{null}\left(\mathrm{C}_{4 k}(a, a)\right)=\operatorname{null}\left(\mathrm{C}_{4 k}^{D 1}(a, a)\right)=2 .
$$

Since $\vec{x}$ and $\vec{y}$ are linearly independent, $\{\vec{x}, \vec{y}\}$ is a basis for $\mathcal{N}\left(\mathrm{C}_{4 k}(a, a)\right)$ and $\mathcal{N}\left(\mathrm{C}_{4 k}^{D 1}(a, a)\right)$.

Theorem 5.5. If $n=0 \bmod 4$ and $a \in \mathbb{R}$, then $\mathrm{C}_{n}^{D 1}(a, a)$ is the Drazin inverse of $\mathrm{C}_{n}(a, a)$.

Proof. Assume $a \neq 0$. Let $n=4 k$ with $k \in \mathbb{N}$. We first prove Condition 2 of Definition 2.2 i.e.,

$$
\left(\mathrm{C}_{n}(a, a)\right)^{2} \mathrm{C}_{4 k}^{D 1}(a, a)=\mathrm{C}_{4 k}(a, a) .
$$

Note that $\mathrm{C}_{4 k}(a, a)=a\left(P+P^{4 k-1}\right)$. By Lemma 5.2 , we conclude that

$$
\mathrm{C}_{4 k}^{D 1}(a, a)=\frac{1}{4 k a} \sum_{t=1}^{2 k} \delta_{2 t-1} P^{2 t-1} .
$$


Since $\left(\mathrm{C}_{4 k}(a, a)\right)^{2}=a^{2}\left(2 I+P^{2}+P^{4 k-2}\right)$, clearly

$$
\begin{aligned}
\left(\mathrm{C}_{4 k}(a, a)\right)^{2} \mathrm{C}_{4 k}^{D 1}(a, a) & \\
& =\frac{a}{4 k}\left(\alpha P+\sum_{t=2}^{2 k-1}\left(\delta_{2 t-3}+2 \delta_{2 t-1}+\delta_{2 t+1}\right) P^{2 t-1}+\beta P^{4 k-1}\right),
\end{aligned}
$$

where $\alpha=\delta_{4 k-1}+2 \delta_{1}+\delta_{3}=3 \delta_{1}+\delta_{3}$ and $\beta=\delta_{4 k-3}+2 \delta_{4 k-1}+\delta_{1}=3 \delta_{1}+\delta_{3}$. Note that

$$
\begin{aligned}
\delta_{1} & =\left((-1)^{\lfloor 1 / 2\rfloor}\lfloor(4 k-1) / 2\rfloor+(-1)^{\lfloor(4 k-1) / 2\rfloor}\lfloor 1 / 2\rfloor\right) \bmod 2 k \\
& =2 k-1
\end{aligned}
$$

and

$$
\begin{aligned}
\delta_{3} & =\left((-1)^{\lfloor 3 / 2\rfloor}\lfloor(4 k-3) / 2\rfloor+(-1)^{\lfloor(4 k-3) / 2\rfloor}\lfloor 3 / 2\rfloor\right) \bmod 2 k \\
& =3-2 k .
\end{aligned}
$$

Hence, $\alpha=\beta=4 k$.

We turn our attention to the sums $\delta_{2 t-3}+2 \delta_{2 t-1}+\delta_{2 t+1}$, for $2 \leq t \leq 2 k-1$. Note that

(1) $\left\lfloor\frac{2 t-3}{2}\right\rfloor=\left\lfloor\frac{2 t-1-2}{2}\right\rfloor=\left\lfloor\frac{2 t-1}{2}\right\rfloor-1$,

(2) $\left\lfloor\frac{2 t+1}{2}\right\rfloor=\left\lfloor\frac{2 t-1+2}{2}\right\rfloor=\left\lfloor\frac{2 t-1}{2}\right\rfloor+1$,

(3) $\left\lfloor\frac{4 k-(2 t-3)}{2}\right\rfloor=\left\lfloor\frac{4 k-(2 t-1)+2}{2}\right\rfloor=\left\lfloor\frac{4 k-(2 t-1)}{2}\right\rfloor+1$, and

(4) $\left\lfloor\frac{4 k-(2 t+1)}{2}\right\rfloor=\left\lfloor\frac{4 k-(2 t-1)-2}{2}\right\rfloor=\left\lfloor\frac{4 k-(2 t-1)}{2}\right\rfloor-1$.

Hence, we can conclude that $-\delta_{2 t-1}=\delta_{2 t-3}=\delta_{2 t+1}$. Therefore, for $2 \leq t \leq 2 k-1$ we have that $\delta_{2 t-3}+2 \delta_{2 t-1}+\delta_{2 t+1}=0$. Thus, $\left(\mathrm{C}_{4 k}(a, a)\right)^{2} \mathrm{C}_{4 k}^{D 1}(a, a)=\mathrm{C}_{4 k}(a, a)$.

Now, by Theorems 2.4 and 5.4 . Condition 3 of Definition 2.2 holds:

$$
\mathrm{C}_{4 k}^{D 1}(a, a) \mathrm{C}_{4 k}(a, a) \mathrm{C}_{4 k}^{D 1}(a, a)=\mathrm{C}_{4 k}^{D 1}(a, a) .
$$

Hence, $\mathrm{C}_{4 k}^{D 1}(a, a)$ is the Drazin inverse of the circulant matrix $\mathrm{C}_{4 k}(a, a)$.

Example 5.6. The Drazin inverse of $\mathrm{C}_{8}(a, a)$ is

$$
\mathrm{C}_{8}^{D}(a, a)=\frac{a^{\#}}{8} \operatorname{Circ}(0,3,0,-1,0,-1,0,3) .
$$

See Figure 5 where for $\mathrm{C}_{8}^{D}(a, a)$ we just draw the directed edges getting out of zero.

Definition 5.7. Let $n=0 \bmod 4$ and $a \in \mathbb{R}$. With $\mathrm{C}_{n}^{D 1 *}(a,-a)$ we denote the circulant matrix

where, for $1 \leq j \leq n-1$,

$$
\frac{a^{\#}}{n} \operatorname{Circ}\left(\delta_{0}^{\prime}, \delta_{1}^{\prime}, 0, \ldots, \delta_{n-1}^{\prime}\right),
$$

with $\delta_{j}$ as in Definition 5.1

$$
\delta_{j}^{\prime}=\operatorname{sgn}(2 j-n)\left|\delta_{j}\right|
$$




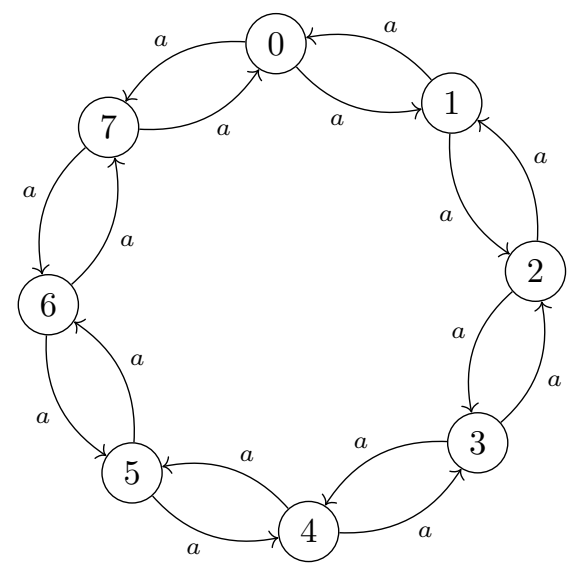

(A) The digraph $D\left(\mathrm{C}_{8}(a, a)\right)$

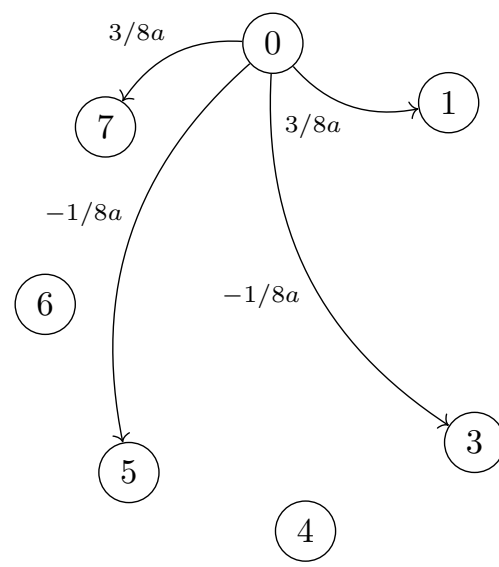

(B) A subdigraph of $D\left(\mathrm{C}_{8}^{D}(a, a)\right)$

Figure 5. The digraphs of a cycle of length 8

In order to prove that $\mathrm{C}_{n}^{D 1 *}(a,-a)$ is the Drazin inverse of $\mathrm{C}_{n}(a,-a)$ we need the following lemma.

Lemma 5.8. Let $n=0 \bmod 4$ and $\delta_{j}^{\prime}$ be as in Definition 5.7. Then $\delta_{j}^{\prime}=-\delta_{n-j}^{\prime}$ for $j \in\{0, \ldots, n-1\}$.

Proof. Let $j \in \mathbb{N} \cup\{0\}$. Then

$$
\begin{aligned}
\delta_{n-j}^{\prime} & =\operatorname{sgn}(2(n-j)-n)\left|\delta_{n-j}\right| \\
& =-\operatorname{sgn}(2 j-n)\left|\delta_{j}\right| \\
& =-\delta_{j}^{\prime} .
\end{aligned}
$$

Theorem 5.9. $\mathcal{N}\left(\mathrm{C}_{4 k}(a,-a)\right)=\mathcal{N}\left(\mathrm{C}_{4 k}^{D 1 *}(a,-a)\right)$.

Proof. Assume $a \neq 0$. The proof is similar to the proof of Theorem 5.4 but using the following vectors $\vec{x}$ and $\vec{y}$ :

$$
x_{i}=\left\{\begin{array}{ll}
1, & \text { if } i=0 \bmod 4 ; \\
1, & \text { if } i=2 \bmod 4 ; \\
0, & \text { otherwise }
\end{array} \quad \text { and } \quad y_{i}= \begin{cases}1, & \text { if } i=1 \bmod 4 \\
1, & \text { if } i=3 \bmod 4 \\
0, & \text { otherwise }\end{cases}\right.
$$

Theorem 5.10. If $n=0 \bmod 4$ and $a \in \mathbb{R}$, then $\mathrm{C}_{n}^{D 1 *}(a,-a)$ is the Drazin inverse of $\mathrm{C}_{n}(a,-a)$.

Proof. Assume $a \neq 0$. Let $n=4 k$ with $k \in \mathbb{N}$. We first prove Condition 2 of Definition 2.2 i.e.,

$$
\left(\mathrm{C}_{4 k}(a,-a)\right)^{2} \mathrm{C}_{4 k}^{D 1 *}(a,-a)=\mathrm{C}_{4 k}(a,-a) .
$$


Note that $\mathrm{C}_{4 k}(a,-a)=a\left(P-P^{4 k-1}\right)$. By Lemma 5.2 we conclude that

$$
\mathrm{C}_{4 k}^{D 1 *}(a,-a)=\frac{1}{4 k a} \sum_{t=1}^{2 k} \delta_{2 t-1}^{\prime} P^{2 t-1} .
$$

Since

clearly

$$
\left(\mathrm{C}_{4 k}(a,-a)\right)^{2}=a^{2}\left(-2 I+P^{2}+P^{4 k-2}\right),
$$

$$
\begin{aligned}
\left(\mathrm{C}_{4 k}(a,-a)\right)^{2} \mathrm{C}_{4 k}^{D 1 *}(a,-a) & \\
& =\frac{a}{4 k}\left(\alpha^{\prime} P+\sum_{t=2}^{2 k-1}\left(\delta_{2 t-3}^{\prime}-2 \delta_{2 t-1}^{\prime}+\delta_{2 t+1}^{\prime}\right) P^{2 t-1}+\beta^{\prime} P^{4 k-1}\right),
\end{aligned}
$$

where $\alpha^{\prime}=\delta_{4 k-1}^{\prime}-2 \delta_{1}^{\prime}+\delta_{3}^{\prime}=-3 \delta_{1}^{\prime}+\delta_{3}^{\prime}$ and $\beta^{\prime}=\delta_{4 k-3}^{\prime}-2 \delta_{4 k-1}^{\prime}+\delta_{1}^{\prime}=-3 \delta_{4 k-1}^{\prime}+$ $\delta_{4 k-3}=3 \delta_{1}^{\prime}-\delta_{3}^{\prime}$.

Note that $\delta_{1}^{\prime}=\operatorname{sgn}(2-4 k)|2 k-1|$ and $\delta_{3}^{\prime}=\operatorname{sgn}(6-4 k)|3-2 k|$. Therefore, if $k=1$, then $\alpha^{\prime}=4$ and $\beta^{\prime}=-4$. If $k>1$, then $\alpha^{\prime}=4 k$ and $\beta^{\prime}=-4 k$. Therefore, all we need to prove is that

$$
\sum_{t=2}^{2 k-1}\left(\delta_{2 t-3}^{\prime}-2 \delta_{2 t-1}^{\prime}+\delta_{2 t+1}^{\prime}\right) P^{2 t-1}=0 .
$$

This is accomplished by showing that $\delta_{2 t-3}^{\prime}-2 \delta_{2 t-1}^{\prime}+\delta_{2 t+1}^{\prime}=0$, for all $t \in$ $\{2, \ldots, 2 k-1\}$.

If $2 \leq t \leq 2 k-1$, since $\delta_{2 t-1}=-\delta_{2 t-3}=-\delta_{2 t+1}$, we have

$$
\begin{aligned}
\delta_{2 t-1}^{\prime} & =\operatorname{sgn}(2(2 t-1)-4 k)\left|\delta_{2 t-1}\right| \\
& =\operatorname{sgn}(4 t-(4 k+2))\left|\delta_{2 t-1}\right|, \\
\delta_{2 t-3}^{\prime} & =\operatorname{sgn}(2(2 t-3)-4 k)\left|\delta_{2 t-3}\right| \\
& =\operatorname{sgn}(4 t-(4 k+6))\left|\delta_{2 t-1}\right|, \\
\delta_{2 t+1}^{\prime} & =\operatorname{sgn}(2(2 t+1)-4 k)\left|\delta_{2 t+1}\right| \\
& =\operatorname{sgn}(4 t-(4 k-2))\left|\delta_{2 t-1}\right| .
\end{aligned}
$$

If $2 \leq t \leq k-1$, then

$$
\begin{aligned}
& \operatorname{sgn}(4 t-(4 k+2))=-1, \\
& \operatorname{sgn}(4 t-(4 k+6))=-1, \\
& \operatorname{sgn}(4 t-(4 k-2))=-1 .
\end{aligned}
$$

Hence, $\delta_{2 t-3}^{\prime}=\delta_{2 t-1}^{\prime}=\delta_{2 t+1}^{\prime}$. Therefore, $\delta_{2 t-3}^{\prime}-2 \delta_{2 t-1}^{\prime}+\delta_{2 t+1}^{\prime}=0$.

If $k+2 \leq t \leq 2 k-1$, then

$$
\begin{aligned}
& \operatorname{sgn}(4 t-(4 k+2))=1, \\
& \operatorname{sgn}(4 t-(4 k+6))=1, \\
& \operatorname{sgn}(4 t-(4 k-2))=1 .
\end{aligned}
$$

Hence, $\delta_{2 t-3}^{\prime}=\delta_{2 t-1}^{\prime}=\delta_{2 t+1}^{\prime}$. Therefore, $\delta_{2 t-3}^{\prime}-2 \delta_{2 t-1}^{\prime}+\delta_{2 t+1}^{\prime}=0$. 
If $t=k$, then

$$
\begin{aligned}
\left|\delta_{2 k-1}\right| & =\left|\left((-1)^{\left\lfloor\frac{2 k-1}{2}\right\rfloor}\left\lfloor\frac{4 k-(2 k-1)}{2}\right\rfloor+(-1)^{\left\lfloor\frac{4 k-(2 k-1)}{2}\right\rfloor}\left\lfloor\frac{2 k-1}{2}\right\rfloor\right) \bmod 2 k\right| \\
& =\left|(-1)^{(k-1)} k+(-1)^{k}(k-1)\right|, \\
\left|\delta_{2 k-3}\right| & =\left|\left((-1)^{\left\lfloor\frac{2 k-3}{2}\right\rfloor}\left\lfloor\frac{4 k-(2 k-3)}{2}\right\rfloor+(-1)^{\left\lfloor\frac{4 k-(2 k-3)}{2}\right\rfloor}\left\lfloor\frac{2 k-3}{2}\right\rfloor\right) \bmod 2 k\right| \\
& =\left|(-1)^{(k-2)}(k+1)+(-1)^{(k+1)}(k-2)\right|, \\
\left|\delta_{2 k+1}\right| & \left.=\mid\left((-1)^{\left\lfloor\frac{2 k+1}{2}\right\rfloor}\left\lfloor\frac{4 k-(2 k+1)}{2}\right\rfloor+(-1)^{\left\lfloor\frac{4 k-(2 k+1)}{2}\right.}\right\rfloor\left\lfloor\frac{2 k+1}{2}\right\rfloor\right) \bmod 2 k \mid \\
& \left.=\mid(-1)^{k}(k-1)+(-1)^{(k-1)} k\right\rfloor .
\end{aligned}
$$

If $k$ is an even number, then

$$
\delta_{2 k-3}^{\prime}-2 \delta_{2 k-1}^{\prime}+\delta_{2 k+1}^{\prime}=-|k+1-k+2|+2|-k+k-1|+|k-1-k|=0 .
$$

If $k$ is an odd number, then

$$
\delta_{2 k-3}^{\prime}-2 \delta_{2 k-1}^{\prime}+\delta_{2 k+1}^{\prime}=-|-k-1+k-2|+2|k-k+1|+|-k+1+k|=0 .
$$

This proves that when $t=k$, we have $\delta_{2 t-3}^{\prime}-2 \delta_{2 t-1}^{\prime}+\delta_{2 t+1}^{\prime}=0$. A similar argument shows that if $t=k+1$, then $\delta_{2 t-3}^{\prime}-2 \delta_{2 t-1}^{\prime}+\delta_{2 t+1}^{\prime}=0$.

Therefore,

$$
\left(\mathrm{C}_{4 k}(a,-a)\right)^{2} \mathrm{C}_{4 k}^{D 1 *}(a,-a)=\frac{a}{4 k}\left(4 k P-4 k P^{4 k-1}\right)=\mathrm{C}_{4 k}(a,-a) .
$$

By Theorems 2.4 and 5.9 ,

$$
\mathrm{C}_{4 k}^{D 1 *}(a,-a) \mathrm{C}_{4 k}(a,-a) \mathrm{C}_{4 k}^{D 1 *}(a,-a)=\mathrm{C}_{4 k}^{D 1 *}(a,-a) .
$$

Hence, $\mathrm{C}_{4 k}^{D 1 *}(a,-a)$ is the Drazin inverse of $\mathrm{C}_{4 k}(a,-a)$.

Example 5.11. For $\mathrm{C}_{8}(a,-a)$, its Drazin inverse is

$$
\mathrm{C}_{8}^{D 1 *}(a,-a)=\frac{a^{\#}}{8} \operatorname{Circ}(0,-3,0,-1,0,1,0,3) .
$$

See Figure 6, where for $\mathrm{C}_{8}^{D 1 *}(a,-a)$ we just draw the directed edges getting out of zero.

\section{CASE 2: $n=2 \bmod 4$}

Definition 6.1. Let $n=2 \bmod 4$ and $a \in \mathbb{R}$. With $\mathrm{C}_{n}^{D 2}(a,-a)$ we denote the circulant matrix

where, for $0 \leq j \leq n-1$,

$$
\frac{2 a^{\#}}{n} \operatorname{Circ}\left(\delta_{0}, \delta_{1}, \ldots, \delta_{n-1}\right),
$$

$$
\delta_{j}= \begin{cases}\frac{2 j-n}{4}, & \text { if } j=1 \bmod 2 \\ 0, & \text { otherwise }\end{cases}
$$




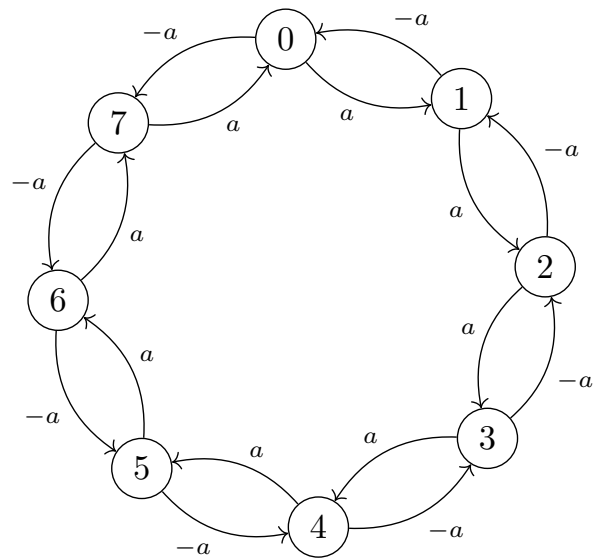

(A) The digraph $D\left(\mathrm{C}_{8}(a,-a)\right)$

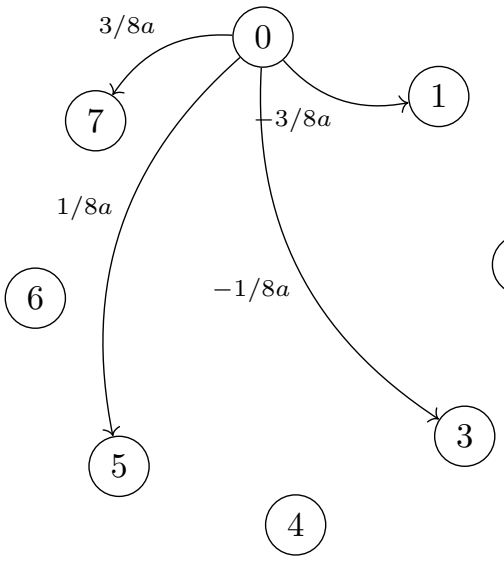

(в) A subdigraph of $D\left(\mathrm{C}_{8}^{D}(a,-a)\right)$

Figure 6 . The digraphs of a cycle of length 8

In order to prove that $\mathrm{C}_{n}^{D 2}(a,-a)$ is the Drazin inverse of $\mathrm{C}_{n}(a,-a)$ we need the following lemma.

Lemma 6.2. Let $n=2 \bmod 4$ and $\delta_{j}$ be as in Definition 6.1. Then $\delta_{j}=-\delta_{n-j}$ for every $j \in\{1, \ldots, n-1\}$.

Proof. If $(n-j)=1 \bmod 2$, then $j=1 \bmod 2$. Therefore,

$$
\delta_{n-j}=\frac{2(n-j)-n}{4}=\frac{n-2 j}{4}=-\delta_{j} .
$$

If $(n-j)=0 \bmod 2$, then $j=0 \bmod 2$. Thus, $\delta_{n-j}=0=-\delta_{j}$.

Theorem 6.3. $\mathcal{N}\left(\mathrm{C}_{4 k+2}(a,-a)\right)=\mathcal{N}\left(\mathrm{C}_{4 k+2}^{D 2}(a,-a)\right)$.

Proof. Assume $a \neq 0$. The proof is similar to the proof of Theorem 5.4, but using the vectors $\vec{x}$ and $\vec{y}$ of the proof of Theorem 5.9

Theorem 6.4. If $n=2 \bmod 4$ and $a \in \mathbb{R}$, then $\mathrm{C}_{n}^{D 2}(a,-a)$ is the Drazin inverse of $\mathrm{C}_{n}(a,-a)$.

Proof. Assume $a \neq 0$. Let $n=4 k+2$ with $k \in \mathbb{N}$. We first prove Condition 2 of Definition 2.2, i.e.,

$$
\left(\mathrm{C}_{4 k+2}(a,-a)\right)^{2} \mathrm{C}_{4 k+2}^{D 2}(a,-a)=\mathrm{C}_{4 k+2}(a,-a) .
$$

Note that $\mathrm{C}_{4 k+2}(a,-a)=a\left(P-P^{4 k+1}\right)$. We have that

$$
\mathrm{C}_{4 k+2}^{D 2}(a,-a)=\frac{1}{(2 k+1) a} \sum_{t=1}^{2 k+1} \delta_{2 t-1} P^{2 t-1} .
$$

Since

$$
\left(\mathrm{C}_{4 k+2}(a,-a)\right)^{2}=a^{2}\left(-2 I+P^{2}+P^{4 k}\right)
$$


clearly

$$
\begin{aligned}
& \left(\mathrm{C}_{4 k+2}(a,-a)\right)^{2} \mathrm{C}_{4 k+2}^{D 2}(a,-a) \\
& =\frac{a}{2 k+1}\left(\alpha^{*} P+\sum_{t=2}^{2 k}\left(\delta_{2 t-3}-2 \delta_{2 t-1}+\delta_{2 t+1}\right) P^{2 t-1}+\beta^{*} P^{4 k+1}\right),
\end{aligned}
$$

where $\alpha^{*}=-\delta_{1}-2 \delta_{1}+\delta_{3}=-3 \delta_{1}+\delta_{3}$ and $\beta^{*}=\delta_{4 k-1}-2 \delta_{4 k+1}+\delta_{1}=3 \delta_{1}-\delta_{3}$.

Since

$$
\delta_{1}=\frac{2-(4 k+2)}{4}=-k \quad \text { and } \quad \delta_{3}=\frac{6-(4 k+2)}{4}=1-k,
$$

we have that $\alpha^{*}=2 k+1$ and $\beta^{*}=-(2 k+1)$. We turn our attention to the sums $\delta_{2 t-3}-2 \delta_{2 t-1}+\delta_{2 t+1}$, for $2 \leq t \leq 2 k$.

We have that $2 t-1,2 t-3$, and $2 t+1$ are odd numbers, and thus

$$
\begin{aligned}
& \delta_{2 t-1}=\frac{2(2 t-1)-(4 k+2)}{4}, \\
& \delta_{2 t-3}=\frac{2(2 t-3)-(4 k+2)}{4}=\delta_{2 t-1}-1, \\
& \delta_{2 t+1}=\frac{2(2 t+1)-(4 k+2)}{4}=\delta_{2 t-1}+1 .
\end{aligned}
$$

Therefore $\delta_{2 t-3}-2 \delta_{2 t-1}+\delta_{2 t+1}=0$. Thus $\left(\mathrm{C}_{4 k+2}(a,-a)\right)^{2} \mathrm{C}_{4 k+2}^{D 2}(a,-a)=$ $\mathrm{C}_{4 k+2}(a,-a)$.

Now, by Theorems 2.4 and 6.3 .

$$
\mathrm{C}_{4 k+2}^{D 2}(a,-a) \mathrm{C}_{4 k+2}(a,-a) \mathrm{C}_{4 k+2}^{D 2}(a,-a)=\mathrm{C}_{4 k+2}^{D 2}(a,-a) .
$$

Hence, $\mathrm{C}_{4 k+2}^{D 2}(a,-a)$ is the Drazin inverse of $\mathrm{C}_{4 k+2}(a,-a)$.

Example 6.5. For $\mathrm{C}_{6}(a,-a)$, its Drazin inverse is

$$
\mathrm{C}_{6}^{D 2}(a,-a)=\frac{a^{\#}}{3} \operatorname{Circ}(0,-1,0,0,0,1) .
$$

See Figure 7. where for $\mathrm{C}_{6}^{D 2}(a,-a)$ we just draw the directed edges getting out of zero.

\section{CASE 3: $n=1 \bmod 2$}

Definition 7.1. Let $n=1 \bmod 2$ and $a \in \mathbb{R}$. With $\mathrm{C}_{n}^{D 3}(a,-a)$ we denote the circulant matrix

where, for $0 \leq j \leq n-1$,

$$
\frac{a^{\#}}{n} \operatorname{Circ}\left(\delta_{0}, \delta_{1}, \ldots, \delta_{n-1}\right)
$$

$$
\delta_{j}= \begin{cases}\frac{j}{2}, & \text { if } j=0 \bmod 2 \\ \frac{j-n}{2}, & \text { otherwise }\end{cases}
$$




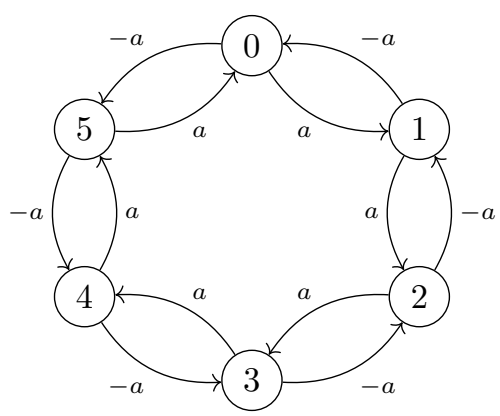

(A) The digraph $D\left(\mathrm{C}_{6}(a,-a)\right)$

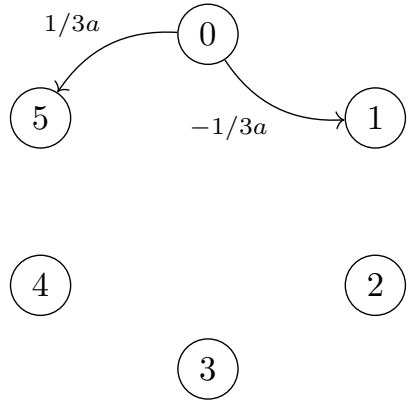

(в) A subdigraph of $D\left(\mathrm{C}_{6}^{D 2}(a,-a)\right)$

Figure 7. The digraphs of a cycle of length 6

In order to prove that $\mathrm{C}_{n}^{D 3}(a,-a)$ is the Drazin inverse of $\mathrm{C}_{n}(a,-a)$ we need the following lemma.

Lemma 7.2. Let $\delta_{j}$ be as in Definition 7.1. Then $\delta_{j}=-\delta_{n-j}$ for every $j \in$ $\{0, \ldots, n-1\}$.

Proof. If $(n-j)=0 \bmod 2$, then $j=1 \bmod 2$. Therefore

$$
\delta_{n-j}=\frac{n-j}{2}=-\delta_{j},
$$

and if $(n-j)=1 \bmod 2$, then $j=0 \bmod 2$. Thus

$$
\delta_{n-j}=\frac{(n-j)-n}{2}=-\delta_{j} .
$$

Theorem 7.3. $\mathcal{N}\left(\mathrm{C}_{2 k+1}(a,-a)\right)=\mathcal{N}\left(\mathrm{C}_{2 k+1}^{D 3}(a,-a)\right)$.

Proof. Assume $a \neq 0$. The proof is similar to the proof of Theorem 5.4 but in this case the null space has dimension 1 . Now, the submatrices of $\mathrm{C}_{2 k+1}(a,-a)$ and $\mathrm{C}_{2 k+1}^{D 3}(a,-a)$, obtained by deleting the first column and the last row, have full rank. The vector $\vec{x}=(1, \ldots, 1)^{T}$ spans both null spaces.

Theorem 7.4. If $n=1 \bmod 2$ and $a \in \mathbb{R}$, then $\mathrm{C}_{n}^{D 3}(a,-a)$ is the Drazin inverse of $\mathrm{C}_{n}(a,-a)$.

Proof. Assume $a \neq 0$. Let $n=2 k+1$ with $k \in \mathbb{N}$. We first prove Condition 2 of Definition 2.2, i.e.,

$$
\left(\mathrm{C}_{2 k+1}(a,-a)\right)^{2} \mathrm{C}_{2 k+1}^{D 3}(a,-a)=\mathrm{C}_{2 k+1}(a,-a) .
$$

Note that $\mathrm{C}_{2 k+1}(a,-a)=a\left(P-P^{2 k}\right)$. We have that

$$
\mathrm{C}_{2 k+1}^{D 3}(a,-a)=\frac{1}{(2 k+1) a} \sum_{t=1}^{2 k} \delta_{t} P^{t} .
$$

Since

$$
\left(\mathrm{C}_{2 k+1}(a,-a)\right)^{2}=a^{2}\left(-2 I+P^{2}+P^{2 k-1}\right),
$$


clearly

$$
\begin{aligned}
\left(\mathrm{C}_{2 k+1}(a,-a)\right)^{2} \mathrm{C}_{2 k+1}^{D 3}(a,-a) & \\
= & \frac{a}{2 k+1}\left(\alpha^{\prime \prime} P+\sum_{t=2}^{2 k-1}\left(\delta_{t-2}-2 \delta_{t}+\delta_{t+2}\right) P^{t}+\beta^{\prime \prime} P^{2 k}\right),
\end{aligned}
$$

where $\alpha^{\prime \prime}=\delta_{2 k}-2 \delta_{1}+\delta_{3}=-3 \delta_{1}+\delta_{3}$ and $\beta^{\prime \prime}=\delta_{2 k-2}-2 \delta_{2 k}+\delta_{1}=3 \delta_{1}-\delta_{3}$.

Note that $\delta_{1}=\frac{1-(2 k+1)}{2}=-k$ and $\delta_{3}=\frac{3-(2 k+1)}{2}=1-k$. Hence, $\alpha^{\prime \prime}=2 k+1$ and $\beta^{\prime \prime}=-(2 k+1)$.

We turn our attention to the sums $\delta_{t-2}-2 \delta_{t}+\delta_{t+2}$, for $2 \leq t \leq 2 k+1$.

If $t=0 \bmod 2$ then $t, t-2$, and $t+2$ are either all odd or all even, and thus

$$
\begin{aligned}
\delta_{t} & =\frac{t}{2}, \\
\delta_{t-2} & =\frac{t-2}{2}=\delta_{t}-1, \\
\delta_{t+2} & =\frac{t+2}{2}=\delta_{t}+1 .
\end{aligned}
$$

Hence, $\delta_{t-2}-2 \delta_{t}+\delta_{t+2}=0$.

Now, if $t=1 \bmod 2$ then $t, t-2$, and $t+2$ are either all odd or all even, and thus

$$
\begin{aligned}
\delta_{t} & =\frac{t-(2 k+1)}{2}, \\
\delta_{t-2} & =\frac{t-2-(2 k+1)}{2}=\delta_{t}-1, \\
\delta_{t+2} & =\frac{t+2-(2 k+1)}{2}=\delta_{t}+1 .
\end{aligned}
$$

Therefore, $\delta_{t-2}-2 \delta_{t}+\delta_{t+2}=0$.

Thus $\left(\mathrm{C}_{2 k+1}(a,-a)\right)^{2} \mathrm{C}_{2 k+1}^{D 3}(a,-a)=\mathrm{C}_{2 k+1}(a,-a)$. Now, by Theorems 2.4 and 7.3

$$
\mathrm{C}_{2 k+1}^{D 3}(a,-a) \mathrm{C}_{2 k+1}(a,-a) \mathrm{C}_{2 k+1}^{D 3}(a,-a)=\mathrm{C}_{2 k+1}^{D 3}(a,-a) .
$$

Hence, $\mathrm{C}_{2 k+1}^{D 3}(a,-a)$ is the Drazin inverse of $\mathrm{C}_{2 k+1}(a,-a)$.

Example 7.5. For $\mathrm{C}_{7}(a,-a)$, its Drazin inverse is

$$
\mathrm{C}_{7}^{D 3}(a,-a)=\frac{a^{\#}}{7} \operatorname{Circ}(0,-3,1,-2,2,-1,3) .
$$

See Figure 8 where for $\mathrm{C}_{7}^{D 3}(a,-a)$ we just draw the directed edges getting out of zero. 


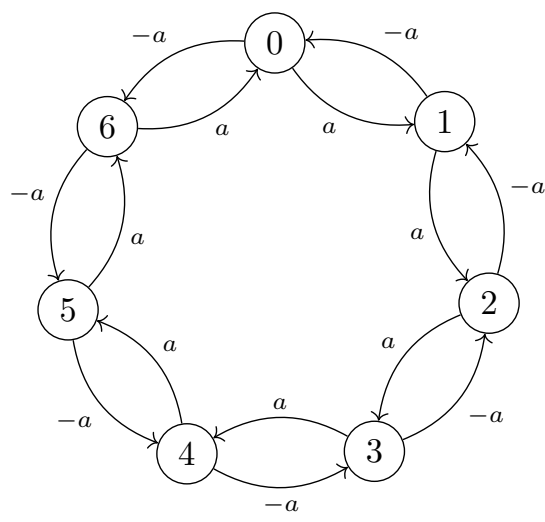

(A) The digraph $D\left(\mathrm{C}_{7}(a,-a)\right)$

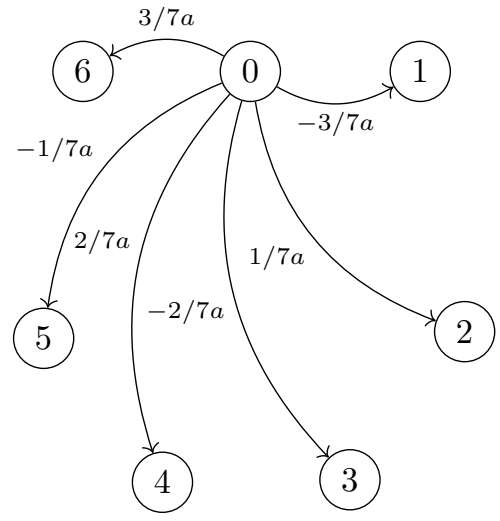

(B) A subdigraph of $D\left(\mathrm{C}_{7}^{D 3}(a,-a)\right)$

Figure 8. The digraphs of a cycle of length 7

\section{REFERENCES}

[1] R. B. Bapat, Graphs and matrices, second edition, Universitext, Springer, London, 2014. MR 3289036

[2] M. Bašić and A. Ilić, On the clique number of integral circulant graphs, Appl. Math. Lett. 22 (2009), no. 9, 1406-1411. MR 2536823

[3] C. L. Bell, Generalized inverses of circulant and generalized circulant matrices, Linear Algebra Appl. 39 (1981), 133-142. MR 0625244

[4] A. Ben-Israel and T. N. E. Greville, Generalized inverses, second edition, CMS Books in Mathematics/Ouvrages de Mathématiques de la SMC, 15, Springer-Verlag, New York, 2003. MR 1987382

[5] A. Bjerhammar, A generalized matrix algebra, Kungl. Tekn. Högsk. Handl. no. 124, Stockholm, 1958. MR 0100608

[6] R. A. Brualdi and D. Cvetković, A combinatorial approach to matrix theory and its applications, Discrete Mathematics and its Applications, CRC Press, Boca Raton, FL, 2009. MR 2453822

[7] A. Carmona, A. M. Encinas, S. Gago, M. J. Jiménez, and M. Mitjana, The inverses of some circulant matrices, Appl. Math. Comput. 270 (2015), 785-793. MR 3406934.

[8] R. H. Chan and T. F. Chan, Circulant preconditioners for elliptic problems, J. Numer. Linear Algebra Appl. 1 (1992), no. 1, 77-101. MR 1169871

[9] I. B. Collings and I. V. L. Clarkson, A low-complexity lattice-based low-PAR transmission scheme for DSL channels, IEEE Trans. Commun. 52 (2004), no. 5, 755-764.

[10] P. J. Davis, Circulant matrices, 2nd edition, AMS Chelsea Publishing, Providence, RI, 1994.

[11] R. Diestel, Graph theory, second edition, Graduate Texts in Mathematics, 173, SpringerVerlag, New York, 2000. MR 1743598

[12] L. Fuyong, The inverse of circulant matrix, Appl. Math. Comput. 217 (2011), no. 21, 84958503. MR 2802259

[13] R. M. Gray, Toeplitz and circulant matrices: A review. Found. Trends Commun. Inform. Theory 2 (2006), no. 3, 155-239.

[14] A. Ilić and M. Bašić, New results on the energy of integral circulant graphs, Appl. Math. Comput. 218 (2011), no. 7, 3470-3482. MR 2851448

[15] D. A. Jaume and R. Sota, Null decomposition and generalized inversion of trees. Unpublished manuscript, 2018. 
[16] Z. Jiang, Y. Gong and Y. Gao, Invertibility and explicit inverses of circulant-type matrices with $k$-Fibonacci and $k$-Lucas numbers, Abstr. Appl. Anal. 2014, Art. ID 238953, 9 pp. MR 3214409

[17] C. Meyer, Matrix analysis and applied linear algebra, Society for Industrial and Applied Mathematics (SIAM), Philadelphia, PA, 2000. MR 1777382.

[18] M. D. Petković and M. Bašić, Further results on the perfect state transfer in integral circulant graphs, Comput. Math. Appl. 61 (2011), no. 2, 300-312. MR 2754138.

[19] B. Radičić, On $k$-circulant matrices (with geometric sequence), Quaest. Math. 39 (2016), no. 1, 135-144. MR 3483362

[20] Ó. Rojo, A new method for solving symmetric circulant tridiagonal systems of linear equations, Comput. Math. Appl. 20 (1990), no. 12, 61-67. MR 1088769.

[21] J. W. Sander, On the kernel of integral circulant graphs, Linear Algebra Appl. 549 (2018), 79-85. MR 3784337

[22] S. R. Searle, On inverting circulant matrices, Linear Algebra Appl. 25 (1979), 77-89. MR 0528714

[23] S.-Q. Shen, J.-M. Cen and Y. Hao, On the determinants and inverses of circulant matrices with Fibonacci and Lucas numbers, Appl. Math. Comput. 217 (2011), no. 23, 9790-9797. MR 2811251

[24] W. So, Integral circulant graphs, Discrete Math. 306 (2006), no. 1, 153-158. MR 2202082.

[25] Y. Tian, Some equalities for generalized inverses of matrix sums and block circulant matrices, Arch. Math. (Brno) 37 (2001), no. 4, 301-306. MR 1879453

[26] J. Wang and C. Dong, Inverse matrix of symmetric circulant matrix on skew field, Int. J. Algebra 1 (2007), no. 9-12, 541-546. MR 2380985

[27] Y. Yazlik and N. Taskara, On the inverse of circulant matrix via generalized $k$-Horadam numbers, Appl. Math. Comput. 223 (2013), 191-196. MR 3116254.

\author{
Andrés M. Encinas \\ Departament de Matemàtiques. Universitat Politècnica de Catalunya. Barcelona, Spain \\ andres.marcos.encinas@upc.edu
}

Daniel A. Jaume $e^{凶}$

Departamento de Matemáticas. Facultad de Ciencias Físico-Matemáticas y Naturales. Universidad Nacional de San Luis. Instituto de Matemáticas Aplicadas de San Luis, IMASL-CONICET, San Luis, Argentina

daniel.jaume.tag@gmail.com

Cristian Panelo

Departamento de Matemáticas. Facultad de Ciencias Físico-Matemáticas y Naturales.

Universidad Nacional de San Luis. San Luis, Argentina

cristian.panelo.tag@gmail.com

\title{
Adrián Pastine
}

Departamento de Matemáticas. Facultad de Ciencias Físico-Matemáticas y Naturales. Universidad Nacional de San Luis. Instituto de Matemáticas Aplicadas de San Luis, IMASL-CONICET, San Luis, Argentina

adrian.pastine.tag@gmail.com

Received: August 16, 2018

Accepted: May 28, 2019 